\title{
Mobile Target Tracking by Networked Uninhabited Autonomous Vehicles via Hospitability Maps
}

\author{
Shreecharan Kanchanavally, Raúl Ordóñez and Jeff Layne
}

\begin{abstract}
An air vehicle detects a target at some position using its own sensor but delays attack. While the target is detected the air vehicle takes several looks at the target in order to classify it. Once the classification of the target is done the same air vehicle or another vehicle attacks it. During the process of classification the target has moved from its initial location. Since the target has moved away where should the UAVs look for it? This is a prediction and a search problem. Prediction uses the past information and search looks for the target in the predicted locations. As time increases the difference between prediction and search becomes blurrier. Therefore the research objective now becomes more challenging. We need to explore innovative modeling and estimation techniques that result in more robust estimation and more robustness to model uncertainties. We can rely on terrain based state prediction to determine the likelihood of the new target position, but the probability density function (pdf) of the target position will propagate, as the target moves. The propagation of the pdf for the target position can be nonlinear, non-Gaussian, and terrain dependent. The effects of such terrains are captured by something called Hospitability maps. A hospitability map provides a likelihood or a "weight" for each point on the terrain's surface for the target to move and maneuver at that location. We introduce the idea of a time varying Hospitability map by making the vehicles searching for the targets part of it.
\end{abstract}

\section{INTRODUCTION}

The idea of multiple uninhabited autonomous vehicles (UAVs) able to adaptively react to their environment and learn about their surroundings while following either an individual or a communal agenda is an intriguing issue. The problem of multi-vehicle coordination and control has been receiving an extraordinary amount of attention during the past few years due to its critical importance for a myriad of applications.

Existing work on multi-vehicle control focuses on receding-horizon planning (that is, optimization methods) and hierarchical structures. The research reported in this paper benefits from previous work that follows the first approach. A receding horizon trajectory planner based on Mixed Integer-Linear Programming (MILP) that is capable

This work was supported with AFRL/AROSR grant No. F33615-01-23154. In addition the work was supported by DAGSI.

Shreecharan Kanchanavally is a student of Electrical and Computer Engineering, University of Dayton, 300 College Park, Dayton, OH 454690226. kanchasc@notes. udayton. edu

Raúl Ordóñez is with Faculty of Electrical and Computer Engineering, University of Dayton, 300 College Park, Dayton, OH 45469-0226. Please send all the correspondence to ordonez@ieee.org

Jeff Layne is with Air Force Research Laboratories, Sensors Directorate, Wright Patterson Air Force Base, Dayton, Ohio 45433-7318. Jeffery. Layne@wpafb.af .mil of planning planar trajectories to a goal constrained by nofly areas, or obstacles, and aircraft dynamics were proposed in [1], [2], [3]. A generalized multi-vehicle formation stabilization problem, free from a leader-follower architecture is defined in [4] and model predictive control (MPC) is applied.

Game theory based cooperative decision making for multi-vehicle include [5], [6] and [7]. Moreover, graph theory is also employed extensively in multi-vehicle coordination. A disjoint path algorithm for reconfiguration of multivehicle was proposed in [8]. A class of triangulated graphs for algebraic representation of formations are introduced to specify a mission cost for a group of vehicles [9], then the obtained optimal control problem is solved using NTG (an optimal control program developed at Caltech). A doublegraph model is used in [10] to treat the string stability as a kind of performance of multi-vehicle system with acyclic formation structures. Moreover, a theoretical explanation of using nearest neighbor rules in coordinating groups of mobile autonomous agents can be found in [11]. The problem of tracking mobile targets using neural network directed Bayes decision rule has been dealt effectively in [12] and [13].

This paper is organized as follows. The next section begins with the formulation of our research problem, followed by development of mathematical model to study the distribution of probability density function using Fokker Planck equation. Surrogate optimization [14], [15] is applied to the cooperative search problem and a specific implementation of the PPP algorithm [20] along with information of the Hmaps for tracking mobile targets is discussed in Section IV. There, Monte Carlo simulations are used to compare these proposed schemes with exhaustive search and the scalability of PPP algorithm is discussed. Finally, Section VI concludes the paper and discusses future work.

\section{Problem Statement}

Here we consider the following situation: an uninhabited autonomous air vehicle (UAV) detects a target using its own sensor, but delays attack. Some time later another UAV or the same UAV is assigned the task of killing the target. By the time the UAV comes to kill it, the target is no longer detected.

In this approach we assume that the target has moved and all the sensors mounted on the UAV provide apt information of the target without loss of generality. Since the target has moved, where should the UAV look for the target? This is a prediction and a search problem. Prediction uses the past 
information (similar to the Kalman filter technique) and the search looks for the target in the predicted locations. As time increases the difference between prediction and search becomes blurrier. Therefore the research objective now becomes more challenging. We need to design and explore innovative modeling techniques that use both prediction and search simultaneously or alternately.

Because the time separation between two looks may be significant, traditional prediction simply based on historic kinematic information will not work. The target kinematics information is diluted quickly as the radius of the possible target location starting at the first look increases. However, the previous kinematics at least provides a center location for the possible target location.

One possible approach is to rely on terrain based state prediction to determine the likelihood of the new target position. The effects of terrain are captured by something known as a hospitability map as explained in [17], [18], [19]. A hospitability map provides a likelihood or a weight for each point on the terrain surface proportional to the ability of a target to move and maneuver at that location. A high hospitability map value denotes that a target can move and maneuver quickly over the corresponding terrain. Likewise, a low hospitability map value indicates that a target cannot easily move over that terrain. The following factors are considered in deriving the hospitability values: slope, surface roughness, transportation, geology, landform, soil, vegetation, hydrology, urban areas and climate.

\section{APPROACH AND MODELING}

In this paper the ground state of the system is found by modeling a diffusion process. We use Fokker-Planck equation and Brownian motion process for modeling.

\section{A. Derivation of Fokker-Planck Equation Using Wiener Process}

We assume that the sensor of the UAV returns nothing other than the position of the target and hence we cannot make any assumptions of its velocity and heading. The best we can do is model the target movement as a diffusion process in all directions following a Wiener process. Consider first the one dimensional case in which the target is moving only in one direction (we shall later extend the discussion to the two dimensional case) where we have

$$
d x \sim \sqrt{d t} N(0,1)
$$

where $d x$ is the white Brownian motion process $d t$ is the time increment and $N(0,1)$ ia a normally distributed random variable. Let $\rho(x, t)$ represent the probability density of the target at time $t$. Once a UAV detects a target this information can be obtained. With this information in mind we can depict the evolution of $\rho(x, t)$ with time as a result of random motion of the target. The equation of change in $\rho(x, t)$ over a time $\Delta t$ is

$$
\rho(x, t+\Delta t)=\int_{-\infty}^{\infty} \rho(x-\Delta x, t) P(\Delta x, \Delta t) d \Delta x(1)
$$

where $P(\Delta x, \Delta t)$ is the probability that the target will move a distance $\Delta x$ in time $\Delta t$. Expanding the above integral using a Taylor series we get

$$
\begin{aligned}
\rho(x, t+\Delta t) \cong & \int\left[\rho(x, t)-\Delta x \frac{\partial \rho}{\partial x}+\frac{1}{2} \Delta x^{2} \frac{\partial^{2} \rho}{\partial x^{2}}\right] \times \\
& P(\Delta x, \Delta t) d \Delta x
\end{aligned}
$$

Since $P$ is a probability density function we have

$$
\int P(\Delta x, \Delta t) d \Delta x=1
$$

Similarly

$$
\int \Delta x P(\Delta x, \Delta t) d \Delta x=0
$$

since it is an average jump, and the variance over a time interval $\Delta t$ is

$$
\int \Delta x^{2} P(\Delta x, \Delta t) d \Delta x=\sigma^{2} \Delta t
$$

Combining the above result we obtain

$$
\rho(x, t+\Delta t)-\rho(x, t)=\frac{\partial \rho}{\partial t} \Delta t=\frac{1}{2} \sigma^{2} \frac{\partial^{2} \rho}{\partial x^{2}} \Delta t
$$

This is correct to order $\Delta t$ so by letting this interval become small we conclude that the density obeys the equation

$$
\frac{\partial \rho}{\partial t}=\frac{1}{2} \sigma^{2} \frac{\partial^{2} \rho}{\partial x^{2}}
$$

Equation (3) represents the Fokker Planck equation in one dimension case. Extending the case to two dimension analysis, with the assumption that the diffusion process is both in the $x$ and $y$ directions and the Brownian process given by

$$
\begin{aligned}
& d x \sim \sqrt{d t} N(0,1) \\
& d y \sim \sqrt{d t} N(0,1)
\end{aligned}
$$

the probability density function is given a by partial differential equation

$$
\frac{\partial \rho}{\partial t}=\frac{\sigma_{x}^{2}}{2} \frac{\partial^{2} \rho}{\partial x^{2}}+\frac{\sigma_{y}^{2}}{2} \frac{\partial^{2} \rho}{\partial y^{2}}
$$

Equation (4) represents the Fokker Planck equation for the two dimensional case.

\section{B. Solution of Fokker-Planck Equation}

This section is mainly concerned with the solution of the Fokker-Planck equation (4). One way of finding the solution to such differential equations is using a finite difference approximation. In this process the continuous time and space variables are discretized such that

$$
\begin{array}{rlrl}
x & =i \Delta x & i & =0,1,2, \ldots, m \\
y & =j \Delta y & j & =0,1,2, \ldots, m \\
t & =k \Delta t & k & =0,1,2, \ldots, m
\end{array}
$$


Let $\rho(i, j, k)$ represent the value of the probability density at $(i \Delta x, j \Delta y, k \Delta t)$ respectively. The solution of (4) is given by

$$
\begin{aligned}
\frac{\rho_{i, j, k+1}-\rho_{i, j, k}}{\Delta t}= & \frac{\sigma_{x}^{2}}{2(\Delta x)^{2}}\left[\rho_{i-1, j, k}-2 \rho_{i, j, k}+\rho_{i+1, j, k}\right] \\
& +\frac{\sigma_{y}^{2}}{2(\Delta y)^{2}}\left[\rho_{i, j-1, k}-2 \rho_{i, j, k}+\rho_{i, j+1, k}\right]
\end{aligned}
$$

solving for $\rho_{i, j, k+1}$ we get

$$
\begin{aligned}
\rho_{i, j, k+1}= & \rho_{i, j, k}+\Delta t\left(\frac{\sigma_{x}^{2}}{2(\Delta x)^{2}}\left[\rho_{i-1, j, k}-2 \rho_{i, j, k}+\rho_{i+1, j, k}\right]\right. \\
& \left.+\frac{\sigma_{y}^{2}}{2(\Delta y)^{2}}\left[\rho_{i, j-1, k}-2 \rho_{i, j, k}+\rho_{i, j+1, k}\right]\right)
\end{aligned}
$$

The above solution is valid for the rectangular region $0<$ $x<a$ and $0<y<b$ and also under the assumption that the initial probability density is known.

\section{Hospitability Maps as Measurement}

Once the propagation of probability density function for the target location is done we apply the hospitablity map $(\mathrm{H}-$ map) at every time instance to constrain the pdf propagation to the regions of higher hospitability. This is done by the following update equation

$$
\rho_{i, j, n}^{+}=\frac{1}{c} \rho_{i, j, n}^{-} H_{i, j}
$$

where the "-" is used to denote the not yet updated pdf and "+" is used to denote the updated pdf, $c$ is a normalizing constant and $H_{i, j}$ is the $i^{t h}, j^{t h}$ cell of the hospitability map. This updated pdf is used in the propagating to the next time instance.

\section{H-MAPS WHEN APPLIED TO PPP SEARCH ALGORITHM}

\section{A. The PPP Search Algorithm}

In this section we apply the H-maps concept developed to the Past Present and Predicted Future (PPP) search algorithm described in [16]. To briefly summarize, the PPP search algorithm is a cooperative search method via surrogate optimization. Surrogate optimization [14], [15] is a non-gradient-based nonlinear programming method that optimizes a function that serves as a surrogate for the objective function (the actual function to minimize). In short, the PPP method assumes that there exists a potential like filed where, to each target corresponds a Gaussian peak. If we call this field $M$, the method performs maximization by letting $M$ be the objective function in the context of surrogate optimization. In this manner, the surrogate $\hat{M}$ that is formed based on the UAV sensor information is an approximation of the real map $M$, where finding each peak then corresponds to finding the static targets.

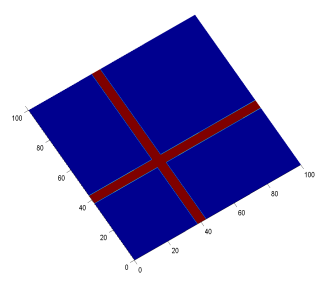

(a)

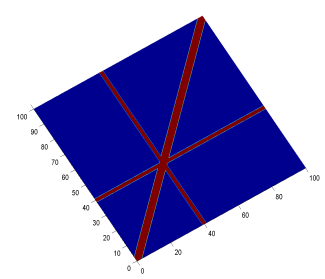

(b)
Fig. 1. Pictorial view of an H-map region. a) With two roads intersecting. b) With three roads intersecting

\section{B. H-maps Applied to PPP Algorithm}

Consider a simple case of an H-map as shown in Figure 1. The square region represents the search area of the networked UAVs. The red color region on this square area represents a road network present in the search area. If a mobile target is found any where in this search area, we assume the only possible way it can take during its motion is the red color region. The important issues to be discussed here are the sensor information given by the UAV on the $\mathrm{H}$-maps and when is this method applicable.

For convenience, we concentrate on discrete time models within a two-dimensional plane, since the setup fits nicely within the simulation environment. We assume whatever constraints exist for vehicles to be not dynamic, but rather kinematic in favor of focusing on high-level mechanisms originating from the group of vehicles. We will assume there are $m$ vehicles and that the $i^{t h}$ one obeys a discrete time kinematic model given by

$$
\begin{aligned}
x_{v_{1}}^{i}(k+1) & =x_{v_{1}}^{i}(k)+d \cos \left(\theta_{v}^{i}(k)\right) \\
x_{v_{2}}^{i}(k+1) & =x_{v_{2}}^{i}(k)+d \sin \left(\theta_{v}^{i}(k)\right) \\
\theta_{v}^{i}(k+1) & =\theta_{v}^{i}(k)+f_{\theta_{v}}\left(u^{i}(k)\right)
\end{aligned}
$$

where $k$ is the discrete time index taking values in the nonnegative integers $\{0,1,2, \ldots\}$ ( $k$ also denotes the number of search steps); $x_{v_{1}}^{i}$ and $x_{v_{2}}^{i}$ are, respectively, the two Cartesian coordinates of $i^{\text {th }}$ vehicle; $d$ is a constant step size; $\theta_{v}^{i}$ is the orientation of the $i^{t h}$ vehicle; $f_{\theta_{v}}$ can be a nonlinear function encoding kinematic restrictions on the vehicles; and $u^{i}$ is the local controller corresponding to the $i^{t h}$ vehicle. For convenience, let $x_{v_{p}}^{i}=\left[x_{v_{1}}^{i}, x_{v_{2}}^{i}\right]^{\top}$, and $x_{v}^{i}=\left[\left(x_{v_{p}}^{i}\right)^{\top}, \theta_{v}^{i}\right]^{\top}$.

Assume that the networked UAV has prior information of the target location and the geographical conditions existing near the target, in particular the H-maps, through a global positioning satellite or another vehicle. Let the search area be a $n \times n$ rectangular map with four UAVs placed at four corners of the rectangle prior to the search ${ }^{1}$. The target environment can be modeled as a two dimensional plane, the upper right quadrant of a cartesian coordinate system with axis $\left(x_{1}, x_{2}\right)$.We assume to have two types of prior

\footnotetext{
${ }^{1}$ Note that the method does not require this initial vehicle arrangement to function, and we do so simply for convenience.
} 
information about the target, in order to handle the static and mobile cases separately. We may set up a Gaussian profile map which is known to all vehicles to represent static targets. The Gaussian profile encodes the possible target locations $x_{s}^{i}=\left[x_{s 1}^{i}, x_{s 2}^{i}\right] \top, i=1, \ldots, n$ offered by the prior information as centers of Gaussian peaks where we assume to know the number $n$ of the static targets,

$$
M_{p}\left(x_{1}, x_{2}, k\right)=\sum_{i=1}^{n} c_{i} \exp \left[\frac{\left(x_{1}-x_{s 1}^{i}\right)^{2}+\left(x_{2}-x_{s 2}^{i}\right)^{2}}{v_{i}^{2}}\right]
$$

We encode the uncertainty of the prior information with the peak width $v_{i}$, and the distance of the real (but unknown) target position of the center of the peak in terms of $v_{i}$. Furthermore, we can intentionally encode the priority level of each target as the height $c_{i}$ of each peak. Thereby, we expect the vehicles to find the most important target first and the whole search performance should coincide with the uncertainty level about the prior information. All the vehicles are provided with sensors which sample a real target map $M_{t}\left(x_{1}, x_{2}\right)$ (Figure $\left.2(\mathrm{~b})\right)$ given by

$M_{t}\left(x_{1}, x_{2}\right)=\left\{\begin{array}{cc}1, & {\left[x_{1}, x_{2}\right]^{\top} \in\left\{\left[x_{t_{1}}^{i}, x_{t_{2}}^{i}\right]^{\top} ; i=1, \ldots, n\right\}} \\ 0, & \text { otherwise }\end{array}\right.$

where $\left\{\left[x_{t 1}^{i}, x_{t 2}^{i}\right] \top ; i=1, \ldots, n\right\}$ is the set of $n$ static target positions and can only obtain two kinds of information: 0 , which means no target, and 1 , means the vehicle found a target. In addition we assume there is only one mobile target initially located somewhere in the area of high hospitability, and we assume to have probabilistic information about this target in the form of a probability density function. Such information could be easily obtained by, e.g., assuming that initially the target locations normally distributed, with the variance equal to the sensor uncertainty and the mean set to the location of the most recent target sighting. We discretize not only the vehicle's movement, but also the proposed map as shown in Figure 2 (a). We will let the vehicles move from cell to cell (i.e., the center of one cell to another) rather than move along a smooth curve, and therefore we will start with the assumption that all vehicles move with constant speed and in discrete time index. Also we assume that maximum turn angles are \pm 135 degrees, i.e., $f_{\theta_{v}}$ becomes a saturation function. Figure 2 (c) illustrates these points: the triangle in the middle cell represents the vehicle's current position with its orientation $\theta_{v}=0$; with the assumptions of constant speed and discrete time index, the vehicle will only move one cell each time index; and with the assumption of maximum angles of turn, the vehicle will only have seven possible cells to go, which are denoted by the circles, and the cross denotes the cell which the vehicle cannot visit since $\theta_{v}=0$. Moreover, we ignore the difference between diagonal step size with vertical or horizontal step size, all denoted as $d$ for convenience. The inter-vehicle communications are instantaneous, noiseless and have unbounded

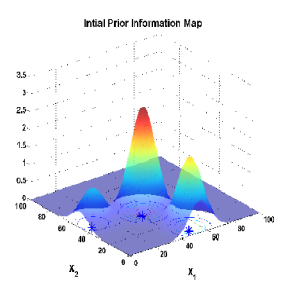

(a)

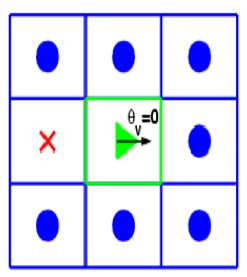

(c)

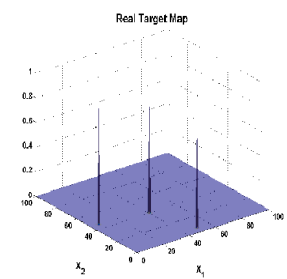

(b)
Fig. 2. (a) Prior information map, (b) Real target map (c) Cell based map assumptions.

communication distance, i.e., we assume perfect communication. These assumptions together with further ones made for the Monte Carlo simulation will be used throughout this paper. Note that this is not a centralized coordination scheme, since if non-ideal communication were considered, each vehicle would have its own version of $M_{p}^{i}$ and would act upon it, sharing as much information as allowed by the communication channel. The level of coordination increases as the quality of the communication channel improves.

All the vehicles in the search area are provided with the information of H-map, $H\left(x_{1}, x_{2}\right)$

$$
H\left(x_{1}, x_{2}\right)=\left\{\begin{array}{cc}
c, & {\left[x_{1}, x_{2}\right]^{\top} \in R d} \\
0, & {\left[x_{1}, x_{2}\right]^{\top} \in B}
\end{array}\right.
$$

where $R d$ represents the the red color regions, $B$ represents the blue color region in the Figure 1 and $c>0$ represents the magnitude of hospitability. As the UAVs start searching for the targets the pdf of the mobile target starts expanding. The probability density distribution $M_{p d}$ is propagated using (6). Each vehicle can update the prior information map (9) with its collected sensor information at time index $k$,

$$
\begin{aligned}
M_{p}\left(x_{1}, x_{2}, k+1\right)= & M_{p}\left(x_{1}, x_{2}, k\right) \cdot M_{s}\left(x_{1}, x_{2}, k\right)+ \\
& \frac{1}{c} M_{p d}\left(x_{1}, x_{2}, k+1\right) \cdot M_{v}\left(x_{1}, x_{2}, k\right)
\end{aligned}
$$

where '.' means element-wise multiplication for matrices and $M_{s}(x, y, k)$ is called sensor matrix, which encodes the current time's sensor information and is defined as follows. Let $S_{1}=\left\{\left[x_{v_{1}}^{i}(k), x_{v_{2}}^{i}(k)\right]^{\top}\right\}$ then

$M_{s}\left(x_{1}, x_{2}, k\right)=\left\{\begin{array}{cc}M_{t}\left(x_{v_{1}}^{i}(k), x_{v_{2}}^{i}(k)\right), & {\left[x_{1}, x_{2}\right]^{\top} \in S_{1}} \\ 1, & \text { otherwise }\end{array}\right.$ 
$M_{v}\left(x_{1}, x_{2}\right)$ is defined as

$$
M_{v}\left(x_{1}, x_{2}\right)=\left\{\begin{array}{cc}
\epsilon, & {\left[x_{1}, x_{2}\right]^{\top} \in\left\{\left[x_{t_{1}}^{i}, x_{t_{2}}^{i}\right]^{\top}\right\}} \\
H\left(x_{1}, x_{2}\right), & \text { otherwise }
\end{array}\right.
$$

where $\epsilon$ is a small value but not equal to zero. In this manner, the H-map changes with time by making the UAV location into regions of low hospitability. This allows us to correctly propagate $M_{p d}$ and keep its integrity as a pdf. More importantly, regions visited by UAV do not remain with a zero probability of the mobile target being there forever. Instead, this probability increases after the UAVs move else where.

\section{Simulation Results}

Assume that a target is located at the middle of the junction shown in Figure 1 a). The experimental set up contains four UAVs initially placed at the corner of the rectangular search map. Figure 3 shows the propagation of probability density function and the zeroing of the pdf at time $T=20,40,60$ and $T=80$ seconds.

The propagation of the pdf is along the Hospitability map (shown in Figure 1) as expected. The spread of the probability density function is similar to the prediction that a human being could make, but in an automated manner.

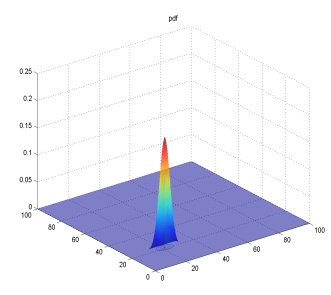

a) $t=20$ seconds

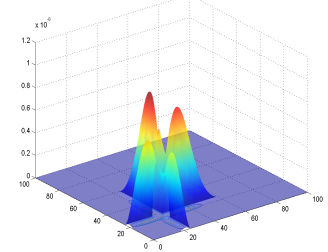

c) $\mathrm{t}=60$ seconds

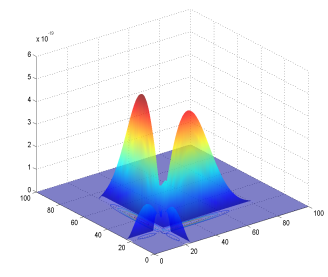

b) $\mathrm{t}=40$ seconds

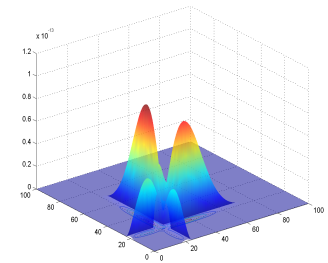

d) $\mathrm{t}=80$ seconds
Fig. 3. Simulation results in H-map region for different time instances

The working of this scenario can be explained as follows. With the target initial location known, the nearest UAV to the target, reaches the target. During its motion if the UAV enters the H-map region it restrict its search only in this region and at the same time if a target is not found it reduces the $\mathrm{H}$-map to $\epsilon$ at that instance, thereby lowering the probability of finding the target there. This generalization can be valid since we are considering that the target moves at a much smaller velocity when compared to the UAV. It is assumed the target moves at one tenth the velocity of the UAV. Once it reaches the presumed target initial location it does not find the target there since it has moved. It then searches at the highest probable point in the H-map During its search the UAV senses for the mobile target, and if not found it reduces the H-map to $\epsilon$ at that instance, and then resets to its previous value as it continues searching. This process of searching for the highest probability point continues until target is found.

As stated earlier the UAVs have a priori information of the initial target location in the form of the pdf $M_{p d}$. In Figure $4 \mathrm{a} \mathrm{UAV}_{1}$ is assigned the task of tracking and killing the moving target. Figure 4 shows the path taken

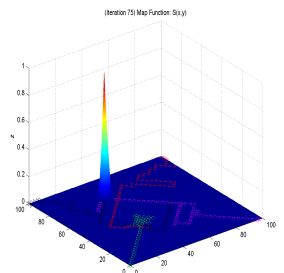

Fig. 4. Path of the UAV

by the UAVs. After a time interval of 28 seconds one of the UAVs in this case $U A V_{1}$ reaches the initial position of the target but could not find it there. Rather than searching through the entire map it starts searching only within the region where the target has the capability to move. This is shown in Figure 4. The other three UAV's are in so-called drive search [20] mode and continuously searching for static targets. This behavior seems to be very natural and sensible from a human point of view. However, the UAV must be able to do it autonomously.

Monte Carlo simulations were run for different numbers of UAVs in so called converging mode [20], for different physical parameters and complexity of H-maps. These results are compared with exhaustive search where we assume to have $n=0$ static targets in order to specifically assess the methods goodness in finding mobile targets. The simulation was run for the road map shown in Figure 1(b) with the target placed at the junction of the roadway. In each test a target is placed randomly at this intersection, and the target moves in a random direction but the motion is continuous. The first column in Table explains the total number of UAVs

\begin{tabular}{|c||c|c|}
\hline No of UAV & Exhaustive search & PPP algorithm \\
\hline \hline 1 & $T=65.16, P=52 \%$ & $T=48.41, P=74 \%$ \\
\hline 2 & $T=16.76, P=86 \%$ & $T=29.83, P=84 \%$ \\
\hline 3 & $T=8.174, P=100 \%$ & $T=20.39, P=94 \%$ \\
\hline
\end{tabular}

TABLE I

WITH 4 UAVS, 1-TARGET AND WIDTH OF ROADWAY EQUAL TO ONE SENSOR FOOTPRINT.

in convergence mode(the total number of UAVs is $m=4$ in all cases). Note that UAV not in convergence mode can be thought of as searching for static targets, therefore not contributing to find a mobile target. The second column 
shows the average time taken to find the target, $T$ (in number of steps), and the probability of finding the target, $P$, computed as $P=\frac{S}{N}$, where $N=50$ is the total number of trials and $S$ is the number of successful trials. when exhaustive search was used. The third column shows the average time taken and the percentage of targets found when PPP algorithm was used to detect moving targets. In this case the width of the H-map is equal to one sensor footprint. Note that in this case exhaustive search performs better when two or three UAVs are in converging mode (in this case, searching exhaustively), since it quickly covers almost the entire possible route taken by the targets. But as the complexity increases this may not be true as shown in Table II. The first, second and third columns represent

\begin{tabular}{|c||c|c|}
\hline No of UAV & Exhaustive search & PPP algorithm \\
\hline \hline 1 & $T=269.64, P=34 \%$ & $T=184.65, P=62 \%$ \\
\hline 2 & $T=188.52, P=68 \%$ & $T=115.12, P=74 \%$ \\
\hline 3 & $T=105.3, P=76 \%$ & $T=79.23, P=88 \%$ \\
\hline
\end{tabular}

TABLE II

WITH 4 UAVS, 1-TARGET AND WIDTH OF ROADWAY EQUAL TO 4 TIMES SENSOR FOOTPRINT.

the same information as explained in Table I with the only difference being that the width of the roads in the H-map is four times the size of the sensor footprint. From the results the following conclusions can be made

1) The greater the width and complexity of the H-map, the greater is the time taken by the UAVs to search and track the mobile targets.

2) The more the number of UAVs in converging mode the less is the time taken to track a mobile target.

3) The smaller the velocity of the target when compared to that of UAV, the less the time taken to track the mobile target.

4) Notice that with one of the UAVs in the converging mode, the average time taken by UAVs in PPP search algorithm is $33.46 \%$ less than that of UAVs in exhaustive search, and also the probability of finding the target in PPP search algorithm is twice the probability of finding the targets in exhaustive search.

5) In the remaining two cases (when two or three UAVs are in converging mode) the average time taken by UAVs in PPP search algorithm is approximately $30 \%$ less than that of UAVs in exhaustive search and the probability of finding the targets in PPP search algorithm is $10 \%$ greater than the probability of finding the targets in exhaustive search.

\section{CONCLUSION}

In this paper we have presented a possible solution to the problem of tracking mobile targets when only a inital position is known. To do so, we use hospitability map to constrain the propagation of a density function denoting the probability of target location. We introduce time-varying hospitability maps, which consider the UAV location as points of low hospitability, and extend the PPP search algorithm to deal with not only static, but also mobile targets. Monte Carlo simulations indicate that the overall scheme performs well, in particular when compared with uncoordinated exhaustive search.

\section{REFERENCES}

[1] J. S. Bellingham, A. Richards, and J. P. How, "Receding horizon control of autonomous aerial vehicles," in Proc. American Control Conf., (Anchorage, Alaska), May 2002.

[2] J. S. Bellingham, M. Tillerson, M. Alighanbari, and J. P. How, "Cooperative path planning for multiple uavs in dynamic and uncertain environments," in Proc. of 41th Conf. Decision Contr., (Las Vegas, NV), 2002.

[3] Y. K. M. Alighanbari and J. P. How, "Coordination and control of multiple uavs with timing constraints and loitering," in Proc. American Control Conf., (Denver, CO), June 2003.

[4] W. B. Dunbar and R. M. Murray, "Model predictive control of coordinated multi-vehicle formations," in Proc. of 41th Conf. Decision Contr., (Las Vegas, NV), 2002.

[5] S. Ganapathy and K. M. Passino, "Agreement strategies for cooperative control of uninhabited autonomous vehicles," in Proc. American Control Conf., (Denver, CO), June 2003.

[6] Y. Liu, M. A. Simaan, and J. J. B. Cruz, "Game theoretic approach to cooperative teaming and tasking in the presence of an adversary," in Proc. American Control Conf., (Denver, CO), June 2003.

[7] Q. Li and S. Payandeh, "Planning for dynamic multi-agent planar manipulation with uncertainty: A game theoretic approach," in Proc. American Control Conf., (Denver, CO), June 2003.

[8] M. E. Broucke, "Disjoint path algorithms for planar reconfiguration of indentical vehicles," in Proc. American Control Conf., (Denver, CO), June 2003.

[9] R. O. Saber, W. B. Dunbar, and R. M. Murray, "Cooperative control of multi-vehicle systems using cost graphs and optimization," in Proc. American Control Conf., (Denver, CO), June 2003.

[10] Z. Jin, W. B. Dunbar, and R. M. Murray, "Stability and performance analysis with double-graph model of vehicle formations," in Proc. American Control Conf., (Denver, CO), June 2003.

[11] A. Jadbabaie, J. Lin, and A. S. Morse, "Coordination of groups of mobile autonomous agents using nearest neighbor rules," IEEE Transactions on Automatic Control, vol. 48, no. 6, pp. 989-1001, 2003.

[12] X. Yu and M. Azimi-Sadjadi, "Neural network directed bayes decision rule for moving target classification," IEEE Transactions on Aerospace and Electronic Systems, vol. 36, pp. 176-188, Jan. 2000.

[13] C. Hue, J.-P. Le Cadre, and P. Perez, "Tracking multiple objects with particle filtering," IEEE Transactions on Aerospace and Electronic Systems, vol. 38, pp. 791- 812, July 2002.

[14] V. Torczon and M. Trosset, "Using approximations to accelerate engineering design optimization," Tech. Rep. ICASE Report No. 9833, NASA/CR, Sept. 1998.

[15] N. Alexandrov, J. J. E. Dennis, R. M. Lewis, and V. Torczon, "A trust region framework for managing the use of approximation models in optimization,' Structural Optimization, vol. 15, pp. 16-23, Feb. 1998.

[16] C. Zhang, R. Ordóñez, and C. Schaumacher, "Multi-vehicle cooperative search with uncertain prior information." Will Appear in AIAA Guidance Navigation and Control Conference, Rhode Islands, August 2004.

[17] J. R. Layne, "A hospitability map approach for estimating a mobile target location." Research Draft.

[18] K. Kastella and C. Kreucher, "Ground target tracking using a multiple model nonlinear filter." Research Draft.

[19] C. Kreucher and K. Kastella, "Multiple model nonlinear filtering for low signal ground target applications." Proceedings of SPIE AeroSense, Signal and Data Processing of Small Targets, Orlando, Florida, April 2001.

[20] C. Zhang and R. Ordóñez, "Decentralized adaptive coordination and control of uninhabited autonomous vehicles via surrogate optimization," in Proc. American Control Conf., (Denver, CO), June 2003. 